\title{
Homotopy analysis for the influence of Navier slip flow in a vertical channel with cross diffusion effects
}

\author{
K. Kaladhar ${ }^{1}$ - E. Komuraiah ${ }^{1}$
}

Received: 8 November 2016/ Accepted: 22 May 2017/Published online: 5 June 2017

(c) The Author(s) 2017. This article is an open access publication

\begin{abstract}
This research is to examine the laminar, incompressible free convective Navier slip flow between vertical plates with cross diffusion effects. This investigation includes the first order chemical reaction also. The resulting equations with boundary conditions are reduced into dimensionless form using suitable transformations. Homotopy analysis method has been applied to solve the system. The influence of emerging parameters on fluid flow quantities have been presented graphically. In addition, the nature of physical quantities are shown in tabular form.
\end{abstract}

Keywords Natural convection - Soret effect · Dufour effect $\cdot$ Chemical reaction $\cdot$ Navier slip $\cdot$ HAM

\section{Introduction}

Combined heat and mass transfer in free convection flow between vertical parallel plates has significant importance in many applications. Kairi and Murthy [1] presented the applications and past theoretical investigations on free convection flow in a channel. Fattahi et al. [2] applied the lattice Boltzmann technique to study the nature of free convection flow of nano fluids. Huelsz and Rechtman [3] also applied the lattice Boltzmann technique for heat transfer in an inclined square cavity due to natural convection. Most recently, Terekhov et al. [4] studied the laminar free convection heat transfer between vertical isothermal plates.

\section{K. Kaladhar}

kaladhar@nitpy.ac.in

1 Department of Mathematics, National Institute of Technology Puducherry, Karaikal 609605, India
Generally, the no-slip condition has been accepted for the fluid over a solid surface boundary condition. At the solid boundary, the general boundary conditions for slip flow has been proposed by Navier [5]. Eegunjobi and Makinde [6] presented the early literature and application of the slip flow in various geometries. Recently, Rundora and Makinde [7] analyzed the influence of Navier slip and variable viscosity through a porous medium with asymmetric convective boundary conditions. Most recently, $\mathrm{Ng}$ [8] investigated the nature of starting flow in channels with boundary slip.

The Soret and Dufour effects are encountered in the areas of chemical engineering, geosciences, etc. The applications and early literature can be seen in [9]. Recently, Srinivasacharya et al. [10] studied the mixed convection flow along a vertical wavy surface with the effects of cross diffusion and variable properties in a porous medium. Most recently, Umar et al. [11] presented the effects of cross diffusions and chemical reaction flow in converging and diverging channels.

Most of the chemical reactions comprise both homogeneous and heterogeneous reactions. The homogeneous reaction takes place in bulk of the fluid, while heterogeneous reaction occurs on some catalytic surfaces. Generally, the interaction between the homogeneous and heterogeneous reactions are very complex and is involved in the production and consumption of reactant species at various rates, both on the catalytic surfaces and within the fluid. The applications and early literature has been reported by Kothandapani and Prakash [12]. Most recently, RamReddy and Pradeepa [13] used the spectral quasi-linearization method to study the effect of homogeneousheterogeneous reactions on non-linear convection flow of micropolar fluid in a porous medium with convective boundary condition. 
In this paper, the natural convection flow in a vertical channel saturated with Navier slip condition has been investigated in the presence of cross diffusions and the rate of chemical reaction. The survey clearly shows that the combined effects with slip flow condition through a vertical channel has not been presented elsewhere. In view of applications and importance, the authors are motivated to take this problem. Homotopy analysis method (HAM) [14-23] has been used for the solution of the present problem. Convergence of the resulting series solution is explained. Then the discussion with respect to the pertinent parameters of the present study on the solutions of velocity, temperature and concentration components.

\section{Mathematical modelling}

We consider two-dimensional free convection fluid flow with Navier slip boundary in a vertical channel. Flow configuration is presented in Fig. 1. All the fluid properties are considered to be constant with the exception that the density in the buoyancy term of the balance of momentum equation. Since the plates are infinitely long, all physical variables depend on $y$ only. The governing two-dimensional steady flow equations can be put into the form:

$v_{y}=0 \Rightarrow v=v_{0}=$ constant

$v_{0} \rho \frac{\partial u}{\partial y}=\rho g\left(\beta_{T}\left(T-T_{1}\right)+\beta_{C}\left(C-C_{1}\right)\right)+\mu \frac{\partial^{2} u}{\partial y^{2}}$

$\rho C_{P} v_{0} \frac{\partial T}{\partial y}=K_{f} \frac{\partial^{2} T}{\partial y^{2}}+\mu\left(\frac{\partial u}{\partial y}\right)^{2}+\frac{D K_{T}}{C_{S} C_{P}} \frac{\partial^{2} C}{\partial y^{2}}$

$v_{0} \frac{\partial C}{\partial y}=D \frac{\partial^{2} C}{\partial y^{2}}+\frac{D K_{T}}{T_{m}} \frac{\partial^{2} T}{\partial y^{2}}-K_{1}\left(C-C_{1}\right)$

with

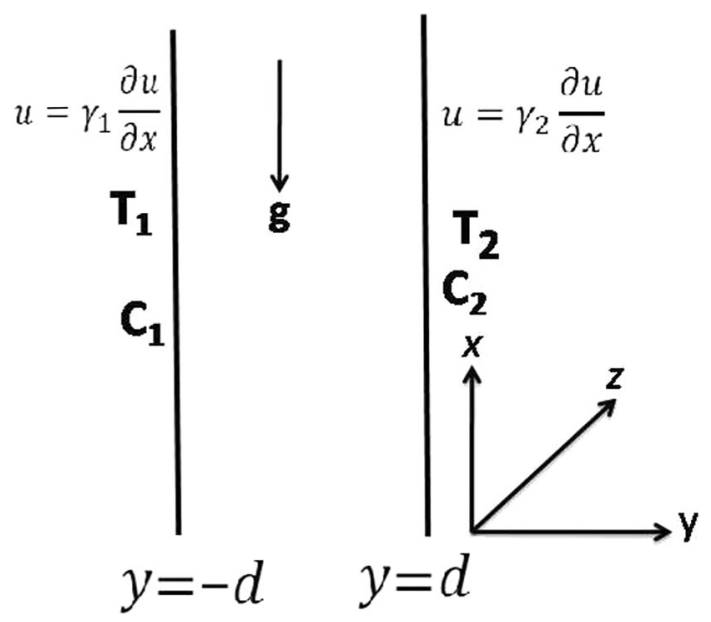

Fig. 1 Physical model and coordinate system.

$$
\begin{array}{lllll}
u=\gamma_{1} \frac{\partial u}{\partial y}, & T=T_{1}, & C=C_{1} & \text { at } & y=-d \\
u=\gamma_{2} \frac{\partial u}{\partial y}, & T=T_{2}, & C=C_{2} & \text { at } & y=d
\end{array}
$$

where the velocity components in $x$ - and $y$ - are $u, v$, respectively, $g$ is the acceleration due to gravity, $\rho$ is the density, $C_{p}$ is the specific heat, $\mu$ is the coefficient of viscosity, $\beta_{T}$ is the coefficient of thermal expansion, $\beta_{C}$ is the coefficient of solutal expansion, $\alpha$ is the thermal diffusivity, $D$ is the mass diffusivity, $K_{T}$ is the coefficient of thermal conductivity, $C_{S}$ is the concentration susceptibility, $T_{m}$ is the mean fluid temperature, $K_{f}$ is the thermal diffusion ratio, $K_{1}$ is the rate of chemical reaction, $\gamma_{1}$ and $\gamma_{2}$ are the slip coefficients at both the plates.

Introducing the following dimensionless variables

$\eta=\frac{y}{d}, u=\frac{v G r}{d} f, \theta=\frac{T-T_{1}}{T_{2}-T_{1}}, \phi=\frac{C-C_{1}}{C_{2}-C_{1}}$

in Eqs. (2)-(4), we obtain the governing dimensionless equations as

$$
\begin{aligned}
& f^{\prime \prime}+\theta+N \phi-\operatorname{Ref}^{\prime}=0 \\
& \theta^{\prime \prime}-\operatorname{RePr} \theta^{\prime}+\operatorname{BrGr}^{2}\left(f^{\prime}\right)^{2}+\operatorname{Pr}_{f} \phi^{\prime \prime}=0 \\
& \phi^{\prime \prime}-\operatorname{ReS} C \phi^{\prime}+\operatorname{ScS} \operatorname{Sr} \theta^{\prime \prime}-K S c \phi=0
\end{aligned}
$$

with

$$
\begin{aligned}
& \eta=-1: f-\beta_{1} f^{\prime}=\theta=\phi=0 \\
& \eta=1: f-\beta_{2} f^{\prime}=0 \quad \text { and } \quad \theta=\phi=1
\end{aligned}
$$

where the primes represents differentiation with respect to $\eta, N=\frac{\beta_{C}\left(C_{2}-C_{1}\right)}{\beta_{T}\left(T_{2}-T_{1}\right)}$ is the buoyancy parameter, $R e=\frac{\rho v_{0} d}{\mu}$ is the Reynolds number, $S c=\frac{v}{D}$ is the Schmidth number, $G r=\frac{g_{a} \beta_{T}\left(T_{2}-T_{1}\right) d^{3}}{v^{2}}$ is the Grashof number, $\operatorname{Pr}=\frac{\mu C_{p}}{K_{f}}$ is the Prandtl number, $B r=\frac{\mu v^{2}}{K_{f} d^{2}\left(T_{2}-T_{1}\right)}$ is the Brinkman number, $K=\frac{K_{1} d^{2}}{v}$ is the chemical reaction parameter, $S_{r}=\frac{D K_{T}\left(T_{2}-T_{1}\right)}{v T_{m}\left(C_{2}-C_{1}\right)}$ is the thermo-diffusion parameter and $D_{f}=\frac{D K_{T}\left(C_{2}-C_{1}\right)}{v C_{S} C_{P}\left(T_{2}-T_{1}\right)}$ is the Dufour number. $\beta_{1}=\frac{\gamma_{1}}{d}$, $\beta_{2}=\frac{\gamma_{2}}{d}$ are the slip parameters.

The skin friction coefficients $\left(C_{f}\right)$, heat $(N u)$ and mass $(S h)$ fluxes at the vertical walls can be given by

$$
\begin{aligned}
\frac{R e^{2}}{G r} C_{f_{1}} & =2 f^{\prime}(-1), \frac{R e^{2}}{G r} C_{f_{2}}=2 f^{\prime}(1) \\
N u_{1,2} & =-\left.\theta^{\prime}(\eta)\right|_{\eta=-1,1}, S h_{1,2}=-\left.\phi^{\prime}(\eta)\right|_{\eta=-1,1}
\end{aligned}
$$


where

$$
C_{f}=\frac{\tau_{w}}{\rho v_{0}^{2}}, N u=\frac{q_{w} d}{K_{f}\left(T_{2}-T_{1}\right)}, S h=\frac{q_{m} d}{D\left(C_{2}-C_{1}\right)}
$$

and

$$
\begin{aligned}
\tau_{w}=\left.\mu \frac{\partial u}{\partial y}\right|_{y= \pm d} ; q_{w} & \left.=-K_{f} \frac{\partial T}{\partial y}\right]_{y= \pm d} \\
q_{m} & \left.=-D \frac{\partial C}{\partial y}\right]_{y= \pm d}
\end{aligned}
$$

Effect of the various parameters involved in the investigation on physical coefficients are discussed in the following section.

\section{Homotopy solution}

For HAM solutions, we choose the initial approximations of $f(\eta), \theta(\eta)$ and $\phi(\eta)$ as follows:

$f_{0}(\eta)=0, \quad \theta_{0}(\eta)=\frac{1+\eta}{2}, \quad \phi_{0}(\eta)=\frac{1+\eta}{2}$

with the auxiliary linear operator

$L=\frac{\partial^{2}}{\partial \eta^{2}} \quad$ such that $L_{1}\left(c_{1} \eta+c_{2}\right)=0$

in which $c_{1}$ and $c_{2}$ are the arbitrary constants. $h_{1}, h_{2}$ and $h_{3}$ (the convergence control parameters) are introduced in zeroth-order deformations as

$$
\begin{aligned}
& (1-p) L\left[f(\eta ; p)-f_{0}(\eta)\right]=p h_{1} N_{1}[f(\eta ; p)] \\
& (1-p) L\left[\theta(\eta ; p)-\theta_{0}(\eta)\right]=p h_{2} N_{2}[\theta(\eta ; p)] \\
& (1-p) L\left[\phi(\eta ; p)-\phi_{0}(\eta)\right]=p h_{3} N_{3}[\phi(\eta ; p)]
\end{aligned}
$$

subject to the boundary conditions

$$
\begin{aligned}
f(-1 ; p)-\beta_{1} f^{\prime}(-1 ; p) & =0, f(1 ; p)-\beta_{2} f^{\prime}(1 ; p)=0 \\
\theta(-1 ; p)=0, \theta(1 ; p) & =1, \phi(-1 ; p)=0, \phi(1 ; p)=1
\end{aligned}
$$

where $p \in[0,1]$ is the embedding parameter and the nonlinear operators $N_{1}, N_{2}$ and $N_{3}$ are defined as:

$$
\begin{aligned}
N_{1}[f(\eta, p), \theta(\eta, p), \phi(\eta, p)]= & f^{\prime \prime}(\eta, p)+\theta(\eta, p) \\
& +N \phi(\eta, p)-\operatorname{Ref}^{\prime}(\eta, p) \\
N_{2}[f(\eta, p), \theta(\eta, p), \phi(\eta, p)]= & \theta^{\prime \prime}(\eta, p)-\operatorname{Re} \operatorname{Pr} \theta^{\prime}(\eta, p) \\
& +\operatorname{BrGr}^{2}\left(f^{\prime}(\eta, p)\right)^{2} \\
& +\operatorname{PrD}_{f} \phi^{\prime \prime}(\eta, p)
\end{aligned}
$$

$$
\begin{aligned}
N_{3}[f(\eta, p), \theta(\eta, p), \phi(\eta, p)]= & \phi^{\prime \prime}(\eta, p)-\operatorname{ReSc} \phi^{\prime}(\eta, p) \\
& +\operatorname{ScSr} \theta^{\prime \prime}(\eta, p)-K \operatorname{KS} \phi(\eta, p)
\end{aligned}
$$

For $p=0$, we have the initial guess approximations

$f(\eta ; 0)=f_{0}(\eta), \theta(\eta ; 0)=\theta_{0}(\eta), \phi(\eta ; 0)=\phi_{0}(\eta)$

When $p=1$, Eqs. (16)-(18) are same as (7)-(9), respectively, therefore, at $p=1$, we get the final solutions

$f(\eta ; 1)=f(\eta), \theta(\eta ; 1)=\theta(\eta), \phi(\eta ; 1)=\phi(\eta)$

Hence, the process of giving an increment to $p$ from 0 to 1 is the process of $f(\eta ; p)$ varying continuously from the initial guess $f_{0}(\eta)$ to the final solution $f(\eta)$ (similar for $\theta(\eta, p)$ and $\phi(\eta, p))$. This kind of continuous variation is called deformation in topology so that we call system of Eqs. (16)-(19), the zeroth-order deformation equation. Next, the $m t h$-order deformation equations follow as

$$
\begin{aligned}
& L_{1}\left[f_{m}(\eta)-\chi_{m} f_{m-1}(\eta)\right]=h_{1} R_{m}^{f}(\eta) \\
& L\left[\theta_{m}(\eta)-\chi_{m} \theta_{m-1}(\eta)\right]=h_{2} R_{m}^{\theta}(\eta) \\
& L\left[\phi_{m}(\eta)-\chi_{m} \phi_{m-1}(\eta)\right]=h_{3} R_{m}^{\phi}(\eta)
\end{aligned}
$$

with the boundary conditions

$$
\begin{aligned}
& f_{m}(-1)=0, \quad f_{m}(1)=0, \quad \theta_{m}(-1)=0 \\
& \theta_{m}(1)=0, \quad \phi_{m}(-1)=0, \quad \phi_{m}(1)=0 \text {, }
\end{aligned}
$$

where

$$
\begin{aligned}
& R_{m}^{f}(\eta)=f^{\prime \prime}+\theta+N \phi-\operatorname{Ref}^{\prime} \\
& R_{m}^{\theta}(\eta)=\theta^{\prime \prime}-\operatorname{Re} \operatorname{Pr} \theta^{\prime}+B r G r^{2} \sum_{n=0}^{m-1} f_{m-1-n}^{\prime} f_{n}^{\prime}+\operatorname{PrD}_{f} \phi^{\prime \prime}
\end{aligned}
$$

$R_{m}^{\theta}(\eta)=\phi^{\prime \prime}-\operatorname{ReSc} \phi^{\prime}+\operatorname{ScSr} \theta^{\prime \prime}-K S c \phi$

for $m$ being integer

$$
\begin{aligned}
& \chi_{m}=0 \quad \text { for } \quad m \leq 1 \\
& =1 \text { for } m>1
\end{aligned}
$$

The initial guess approximations $f_{0}(\eta), \theta_{0}(\eta)$ and $\phi_{0}(\eta)$, the linear operators $L$ and the auxiliary parameters $h_{1}, h_{2}$ and $h_{3}$ are assumed to be selected such that Eqs. (16)-(19) have solution at each point $p \in[0,1]$ and also with the help of Taylor's series and due to Eq. (23); $f(\eta ; p), \theta(\eta ; p)$ and $\phi(\eta ; p)$ can be expressed as

$f(\eta ; p)=f_{0}(\eta)+\sum_{m=1}^{\infty} f_{m}(\eta) p^{m}$ 
$\theta(\eta ; p)=\theta_{0}(\eta)+\sum_{m=1}^{\infty} \theta_{m}(\eta) p^{m}$

$\phi(\eta ; p)=\phi_{0}(\eta)+\sum_{m=1}^{\infty} \phi_{m}(\eta) p^{m}$

in which $h_{1}, h_{2}$ and $h_{3}$ are chosen in such a way that the series (33)-(35) are convergent [15] at $p=1$. Therefore, we have from (24) that

$$
\begin{aligned}
& f(\eta)=f_{0}(\eta)+\sum_{m=1}^{\infty} f_{m}(\eta) \\
& \theta(\eta)=\theta_{0}(\eta)+\sum_{m=1}^{\infty} \theta_{m}(\eta) \\
& \phi(\eta)=\phi_{0}(\eta)+\sum_{m=1}^{\infty} \phi_{m}(\eta)
\end{aligned}
$$

for which we presume that the initial guesses to $f, \theta$ and $\phi$, the auxiliary linear operators $L$ and the non-zero auxiliary parameters $h_{1}, h_{2}$ and $h_{3}$ are so properly selected that the deformation $f(\eta, p), \theta(\eta, p)$ and $\phi(\eta, p)$ are smooth enough and their mth-order derivatives with respect to $p$ in Eqs. (36)-(38) exist and are given, respectively, by $f_{m}(\eta)=\left.\frac{1}{m !} \frac{\partial^{m} f(\eta ; p)}{\partial p^{m}}\right|_{p=0}, \quad \theta_{m}(\eta)=\left.\frac{1}{m !} \frac{\partial^{m} \theta(\eta ; p)}{\partial p^{m}}\right|_{p=0} \quad$ and $\phi_{m}(\eta)=\left.\frac{1}{m !} \frac{\partial^{m} \phi(\eta ; p)}{\partial p^{m}}\right|_{p=0}$. It is clear that the convergence of Taylor series at $p=1$ is a prior assumption, whose justification is provided via a theorem [25], so that the system in (36)-(38) holds true. The formulae in (36)-(38) provide us with a direct relationship between the initial guesses and the exact solutions. All the effects of interaction of the magnetic field as well as of the heat transfer, Hall and Ion effects and couple stress flow field can be studied from the exact formulas (36)-(38). Moreover, a special emphasize should be placed here that the mth-order deformation system (25)-(28) is a linear differential equation system with the auxiliary linear operators $L$ whose fundamental solution is known.

\section{Convergence of the HAM solution}

The expressions for $f, \theta$ and $\phi$ contain the auxiliary parameters $h_{1}, h_{2}$ and $h_{3}$. As pointed out by Liao [14], the convergence and the rate of approximation for the HAM solution strongly depend on the values of auxiliary parameter $h$. For this purpose, $h$-curves are plotted to choose $h_{1}, h_{2}$ and $h_{3}$ in such a manner that the solutions (33)-(35) ensure convergence [14]. Here to see the admissible values of $h_{1}, h_{2}$ and $h_{3}$, the $h$-curves are plotted for 15th-order of approximation in Figs. 2, 3 and 4 by

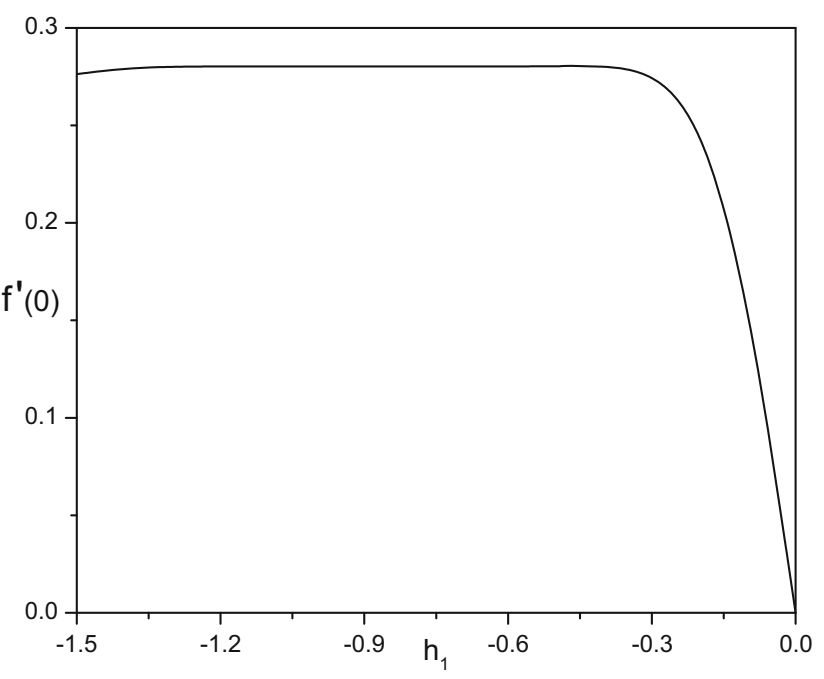

Fig. 2 The $h$ curve of $f(\eta)$

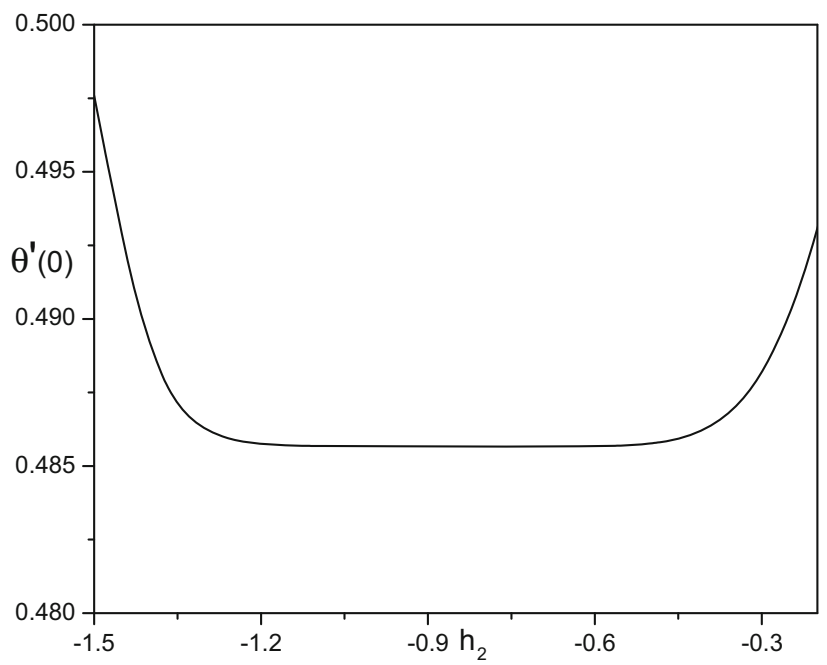

Fig. 3 The $h$ curve of $\theta(\eta)$

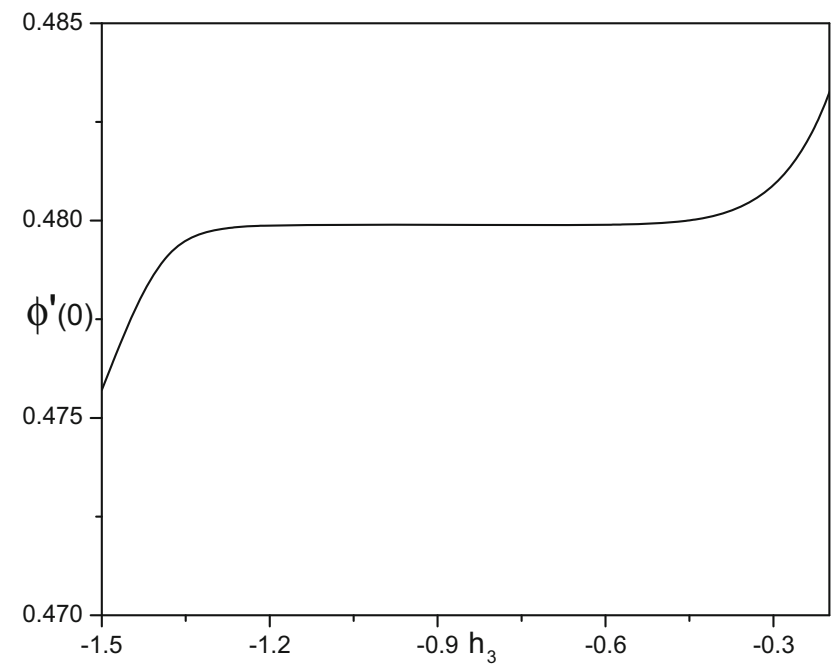

Fig. 4 The $h$ curve of $\phi(\eta)$ 
taking the values of the parameters $N=2, \operatorname{Re}=2, \operatorname{Pr}=$ $0.71, S c=0.22, B r=0.5, G r=0.5, S r=0.5, D f=0.5$, $K=1.0$. It is clearly noted from Fig. 2 that the range for the admissible values of $h_{1}$ is $-1.25<h_{1}<-0.6$. From Fig. 3 , it can be seen that the $h$-curve has a parallel line segment that corresponds to a region $-1.15<h_{2}<-0.65$. Figure 4 depicts that the admissible value of $h_{3}$ are $-1.2<h_{3}<-0.6$. A wide valid zone is evident in these figures ensuring convergence of the series. To choose optimal value of auxiliary parameters, the average residual errors (see Ref. [15] for more details) are defined as

$$
\begin{aligned}
& E_{f, m}=\frac{1}{2 K} \sum_{i=-K}^{K}\left(N_{1}\left[\sum_{j=0}^{m} f_{j}(i \triangle t)\right]\right)^{2} \\
& E_{\theta, m}=\frac{1}{2 K} \sum_{i=-K}^{K}\left(N_{2}\left[\sum_{j=0}^{m} \theta_{j}(i \triangle t)\right]\right)^{2} \\
& E_{\phi, m}=\frac{1}{2 K} \sum_{i=-K}^{K}\left(N_{3}\left[\sum_{j=0}^{m} \phi_{j}(i \triangle t)\right]\right)^{2}
\end{aligned}
$$

where $\Delta t=1 / K$ and $K=5$. At different order of approximations $(m)$, minimum of average residual errors are shown in Tables 1, 2 and 3. It is clear from Table 1 that the average residual error for $f$ is minimum at $h_{1}=-0.93$. It can be seen from Table 2 that the minimum of average residual error for $\theta$ attains at $h_{2}=-0.93$. Table 3 depicts that at $h_{3}=-0.93, E_{\phi}$ attains minimum. Therefore, the optimum values of convergence control parameters are taken as $h_{1}=-0.93, h_{2}=-0.93, h_{3}=-0.93$.

To see the accuracy of the solutions, the residual errors are defined for the system as

$$
\mathrm{RE}_{f}=f_{n}^{\prime \prime}(\eta)+\theta_{n}(\eta)+N \phi_{n}(\eta)-\operatorname{Ref}_{n}^{\prime}(\eta)
$$

Table 1 Optimal value of $h_{1}$ at different order of approximations

\begin{tabular}{lll}
\hline Order & Optimal of $h_{1}$ & Minimum of $E_{m}$ \\
\hline 10 & -0.93 & $1.30 \times 10^{-10}$ \\
15 & -0.93 & $5.45 \times 10^{-16}$ \\
20 & -0.93 & $1.36 \times 10^{-18}$ \\
\hline
\end{tabular}

Table 2 Optimal value of $h_{2}$ at different order of approximations

\begin{tabular}{lll}
\hline Order & Optimal of $h_{2}$ & Minimum of $E_{m}$ \\
\hline 10 & -0.94 & $2.01 \times 10^{-10}$ \\
15 & -0.93 & $4.33 \times 10^{-14}$ \\
20 & -0.93 & $1.44 \times 10^{-16}$ \\
\hline
\end{tabular}

Table 3 Optimal value of $h_{3}$ at different order of approximations

\begin{tabular}{lll}
\hline Order & Optimal of $h_{3}$ & Minimum of $E_{m}$ \\
\hline 10 & -0.93 & $1.50 \times 10^{-11}$ \\
15 & -0.93 & $5.63 \times 10^{-15}$ \\
20 & -0.93 & $2.10 \times 10^{-17}$ \\
\hline
\end{tabular}

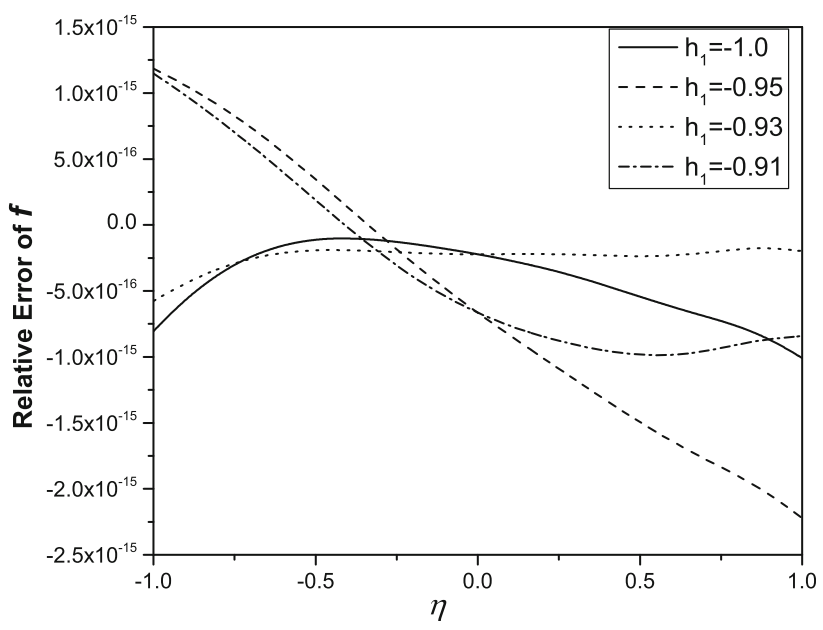

Fig. 5 Residual error of $f(\eta)$ when $\beta_{h}=2, \beta_{i}=2, \alpha=0.5, H a=5$

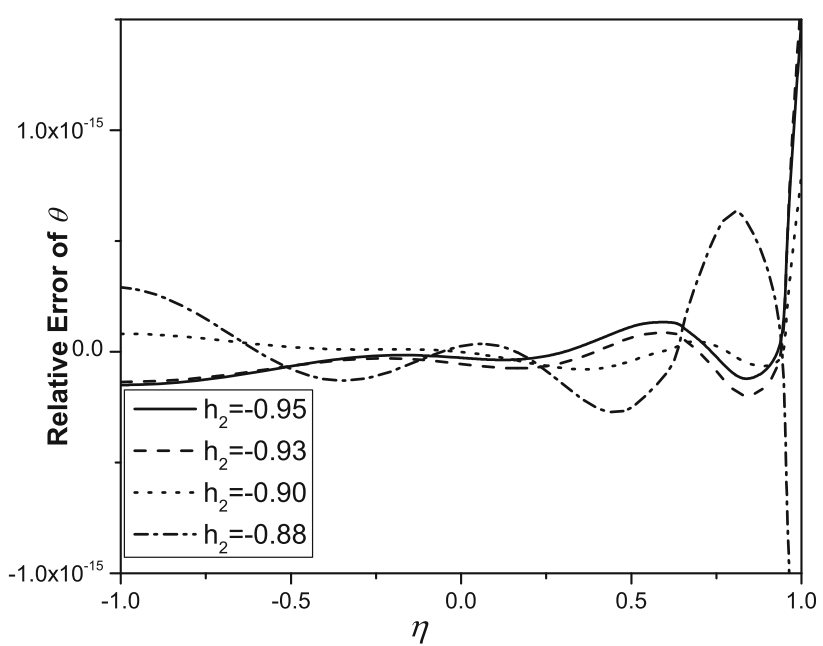

Fig. 6 Residual error of $\theta(\eta)$ when $\beta_{h}=2, \beta_{i}=2, \alpha=0.5, H a=5$

$$
\begin{aligned}
\operatorname{RE}_{\theta}= & \theta_{n}^{\prime \prime}(\eta)-\operatorname{RePr} \theta_{n}^{\prime}(\eta) \\
& +B r G r^{2}\left(f_{n}^{\prime}(\eta)\right)^{2}+\operatorname{PrD}_{f} \phi_{n}^{\prime \prime}(\eta) \\
\operatorname{RE}_{\theta}= & \phi_{n}^{\prime \prime}(\eta)-\operatorname{ReSc} \phi_{n}^{\prime}(\eta) \\
& +\operatorname{ScSr} \theta_{n}^{\prime \prime}(\eta)-K S c \phi_{n}(\eta)
\end{aligned}
$$

where $f_{n}(\eta), \theta_{n}(\eta)$ and $\phi_{n}(\eta)$ are the HAM solutions for $f(\eta), \theta(\eta)$ and $\phi(\eta)$, respectively. For optimality of the 


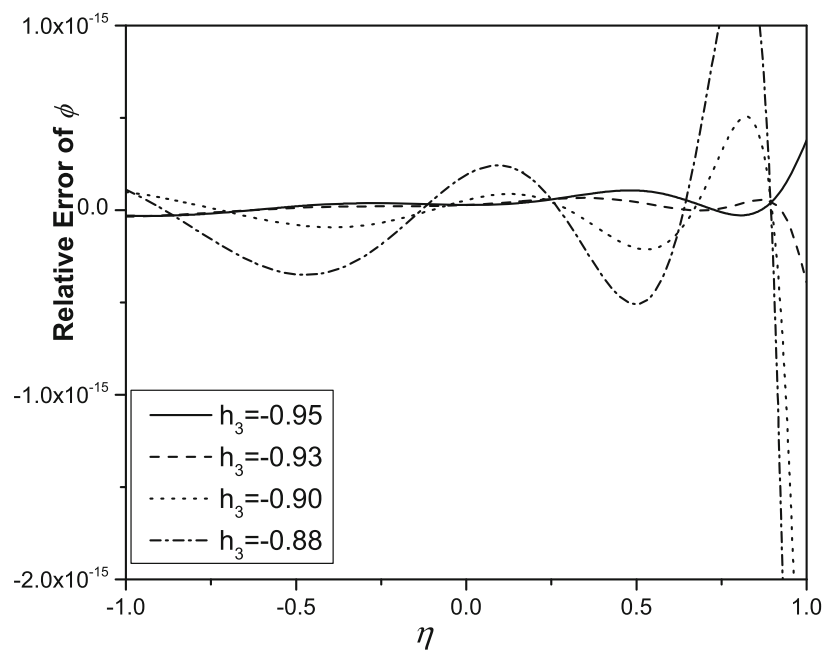

Fig. 7 Residual error of $\phi(\eta)$ when $\beta_{h}=2, \beta_{i}=2, \alpha=0.5, \mathrm{Ha}=5$

convergence control parameters, residual error [24] for different values of $h$ in the convergence region displayed in Figs. 5, 6 and 7. We examine that $h_{1}=-0.93, h_{2}=$ $-0.93, h_{3}=-0.93$ gives a better solution. Table 4 establishes the convergence of the obtained series solution. It is found from the above observations that the series given by (33)-(35) converge in the whole region of $\eta$ when $h_{1}=-0.93, h_{2}=-0.93, h_{3}=-0.93$

To pursue the convergence of the HAM solutions to the exact ones, the graphs for the ratio (following the recent work of [25])

$\beta_{f}=\left|\frac{f_{m}(h)}{f_{m-1}(h)}\right|, \beta_{\theta}=\left|\frac{\theta_{m}(h)}{\theta_{m-1}(h)}\right|, \beta_{\phi}=\left|\frac{\phi_{m}(h)}{\phi_{m-1}(h)}\right|$

against the number of terms $m$ in the homotopy series is presented in Figs. 8, 9 and 10. Figures strongly indicate that a finite limit of $\beta$ will be attained in the limit of $m \rightarrow \infty$, which will remain less than or equals to unity. The velocity and temperature solutions seem to converge in an oscillatory manner requiring more terms in the homotopy series. Thus, the convergence to the exact solution is assured by the HAM method.

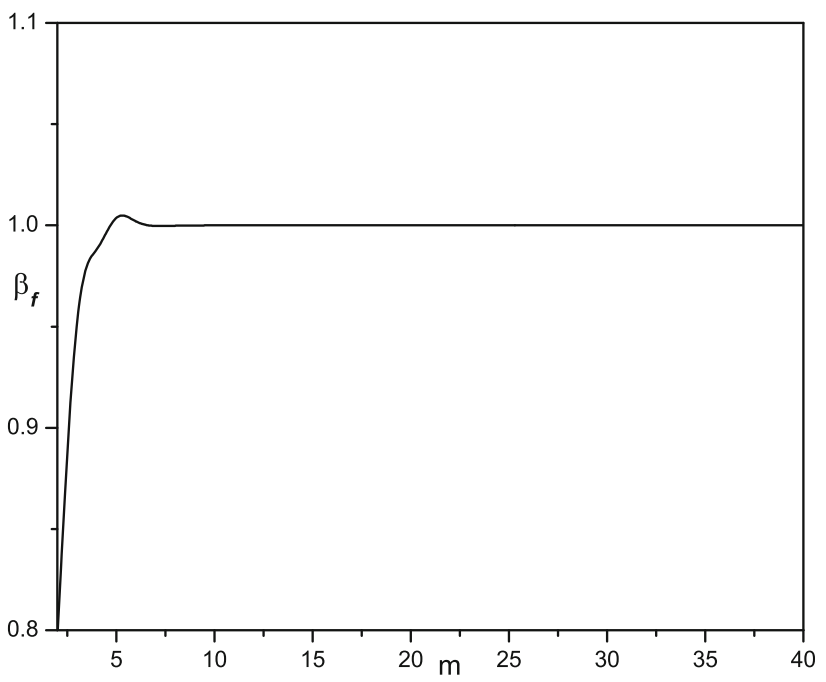

Fig. 8 The ratio $\beta_{f}$ from the theorem to reveal the convergence of the HAM solutions

\section{Discussion of results}

In the absence of Brinkman $(\mathrm{Br}=0)$ number, the system of Eqs. (7)-(9) reduces to the linear system of equations for which exact solution exist. Comparison between analytical solution and HAM solution is shown in Table 5. The comparisons are found to be in a very good agreement. Therefore, the HAM code can be used with great confidence to study the problem considered in this paper.

The profiles of velocity $(f(\eta))$, temperature $(\theta(\eta))$ and concentration $(\phi(\eta))$ are computed and presented through plots in Figs. 11, 12, 13, 14, 15, 16, 17, 18, 19, 20 and 21 with different values of $\beta_{1}, \beta_{2}, S r, D_{f}, K$. Computations were carried out by fixing the parameters $\operatorname{Pr}=0.71, N=$ 2, $B r=0.5, D a=0.5, R e=2, S c=0.22, G r=0.5 \quad$ to analyze the effects of the emerging parameters $\beta_{1}, \beta_{2}, S r$, $D_{f}$ and $K$.

Figure 11 presents the influence of the slip flow parameter $\beta_{1}$ on $f(\eta)$. It is clear that increase in the parameter $\beta_{1}$ leads to increase the flow velocity $f(\eta)$ at the injection wall and there is slight reverse trend can be found at the terminal wall. Figure 12 shows that the effect of $\beta_{2}$
Table 4 Convergence of HAM solutions for different order of approximations

\begin{tabular}{llll}
\hline Order & $f(0)$ & $\theta(0)$ & $\phi(0)$ \\
\hline 4 & 0.4910169474433473893 & 0.387920461619234213657136 & 0.428633469889216020427361 \\
8 & 0.4947894544255234236 & 0.378481745766847297706702 & 0.424271364200980527767338 \\
12 & 0.4948135285227568114 & 0.378404924235389153211848 & 0.424337509824766393328840 \\
16 & 0.4948137463089264307 & 0.378403051384050246849151 & 0.424339108852924445123161 \\
20 & 0.4948137528096351068 & 0.378403073998202584356433 & 0.424339130178531704167978 \\
24 & 0.4948137530282150863 & 0.378403078729212125985981 & 0.424339129132763810471734 \\
28 & 0.4948137530306036798 & 0.378403079015738519907694 & 0.424339129022152340152007 \\
30 & 0.4948137530306036798 & 0.378403079015738519907694 & 0.424339129022152340152007 \\
\hline
\end{tabular}




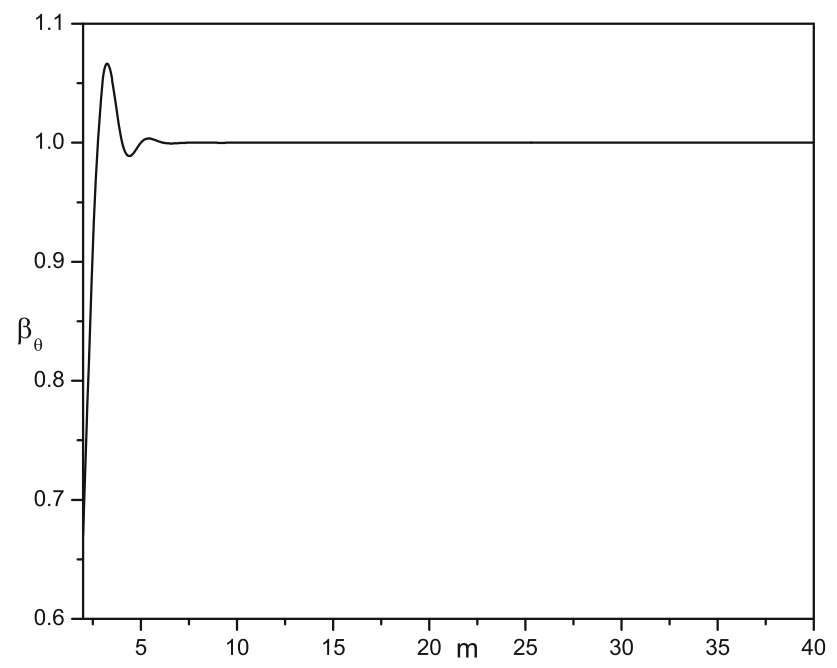

Fig. 9 The ratio $\beta_{\theta}$ from the theorem to reveal the convergence of the HAM solutions

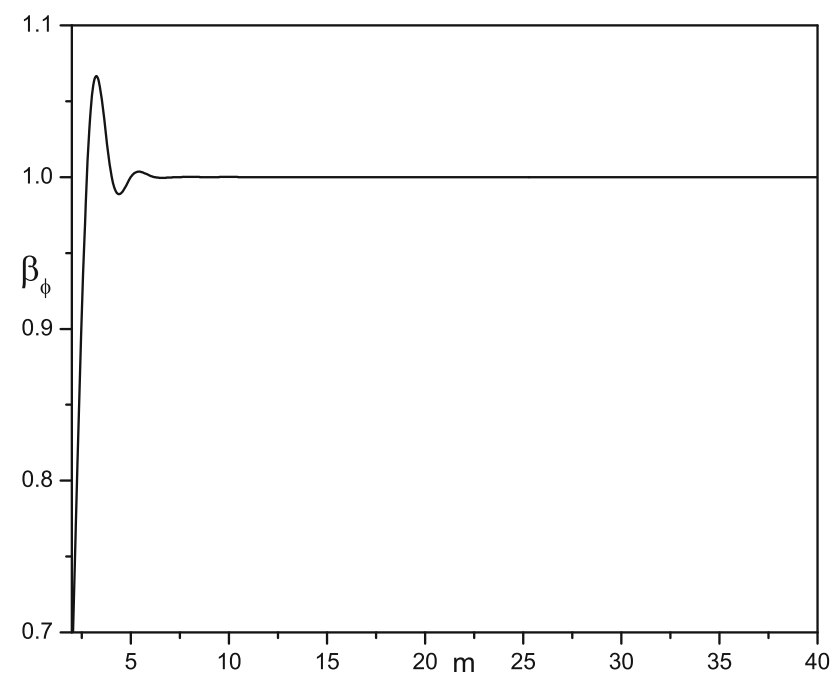

Fig. 10 The ratio $\beta_{\phi}$ from the theorem to reveal the convergence of the HAM solutions

on the flow velocity $f(\eta)$. It can be seen from this figure that as $\beta_{2}$ increases the flow velocity decreases negotiably at the initial wall but appreciable decrease in the velocity is noticed at the suction wall.

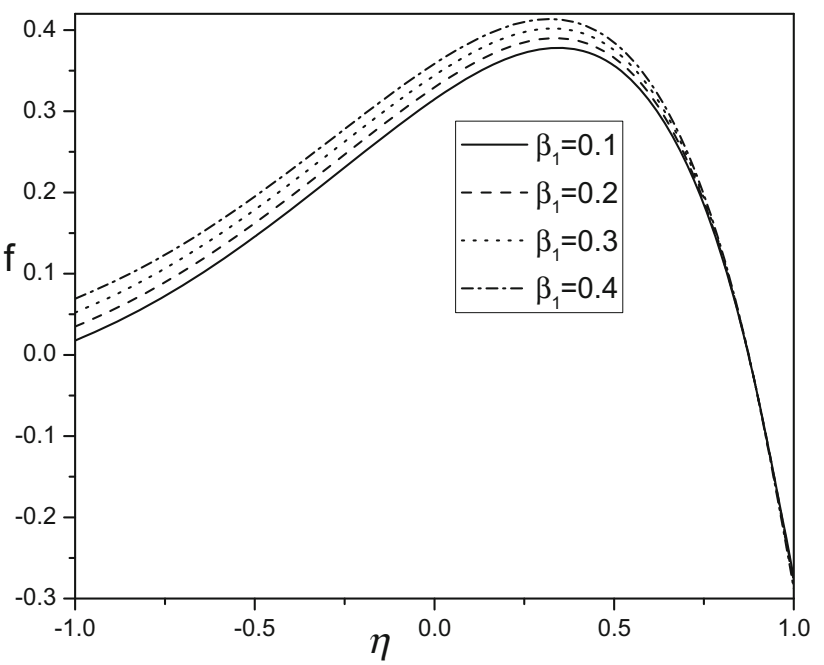

Fig. 11 Variation $f(\eta)$ with $\beta_{1}$ when $\beta_{2}=0.1, S r=0.5$, $D_{f}=0.5, K=1.0$

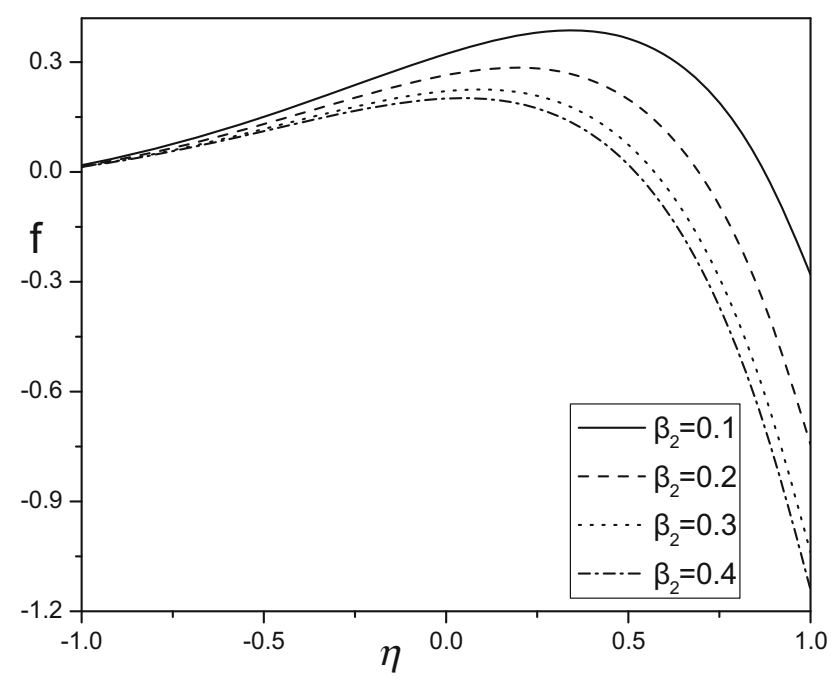

Fig. 12 Variation $f(\eta)$ with $\beta_{2}$ when $\beta_{1}=0.1, S r=0.5, D_{f}=0.5$, $K=1.0$

The effect of Soret parameter on velocity, temperature and concentration profiles are presented in Figs. 13, 14 and 15. It is clear from Fig. 13 that the higher values of Soret
Table 5 Comparison of exact solution $(\mathrm{Br}=0)$ with HAM solution

\begin{tabular}{|c|c|c|c|c|c|c|}
\hline \multirow[b]{2}{*}{$\eta$} & \multicolumn{2}{|l|}{$f$} & \multicolumn{2}{|l|}{$\theta$} & \multicolumn{2}{|l|}{$\phi$} \\
\hline & Analytical & HAM & Analytical & HAM & Analytical & HAM \\
\hline-1 & 0.026479 & 0.026479 & 0 & 0 & 0 & 0 \\
\hline-0.6 & 0.148563 & 0.148563 & 0.112667 & 0.112667 & 0.173056 & 0.173056 \\
\hline-0.2 & 0.275758 & 0.275758 & 0.257042 & 0.257042 & 0.359029 & 0.359029 \\
\hline 0.2 & 0.345735 & 0.345735 & 0.443246 & 0.443246 & 0.558629 & 0.558629 \\
\hline 0.6 & 0.254633 & 0.254633 & 0.684836 & 0.684836 & 0.772288 & 0.772288 \\
\hline 1 & -0.16612 & -0.16612 & 1 & 1 & 1 & 1 \\
\hline
\end{tabular}




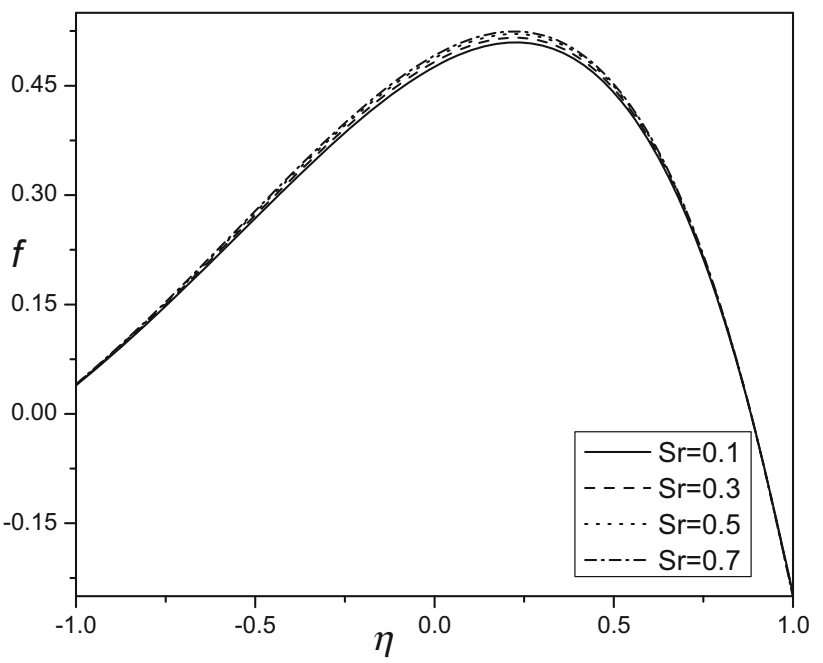

Fig. 13 Soret effect on $f(\eta)$ when $\beta_{1}=0.1, \beta_{2}=0.1$, $D_{f}=0.5, K=1.0$

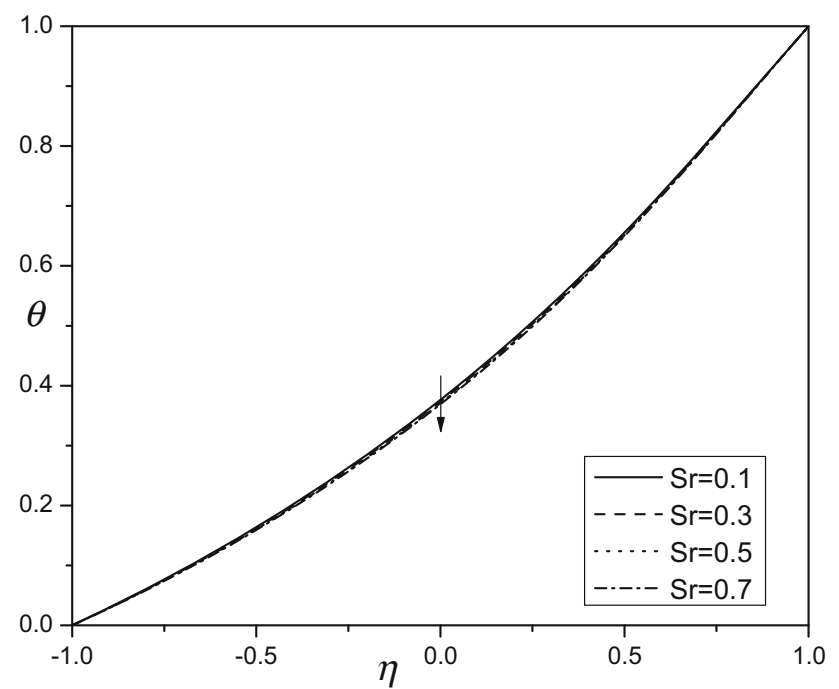

Fig. 14 Soret effect on $\theta(\eta)$ when $\beta_{1}=0.1, \beta_{2}=0.1, D_{f}=$ $0.5, K=1.0$

number $S r$ increases the velocity of the flow. Since the values of $S_{r}$ increases due to either an decrease in the temperature difference or increase in the concentration difference, with this lowest peak of the flow velocity compatible with the lowest Soret number. Figure 14 shows the influence of $S r$ on $\theta$. It is observed that the temperature decreases as $S r$ increases. The nature of $\phi$ with the influence of $S r$ is shown in Fig. 15. It is noted that the dimensionless concentration increases as an increase in the Soret number. The results clearly exhibits that the flow field is significantly influenced by the Soret number.

The effect of Dufour number $\left(D_{f}\right)$ on dimensionless velocity can be seen in Fig. 16. It is clear that the velocity

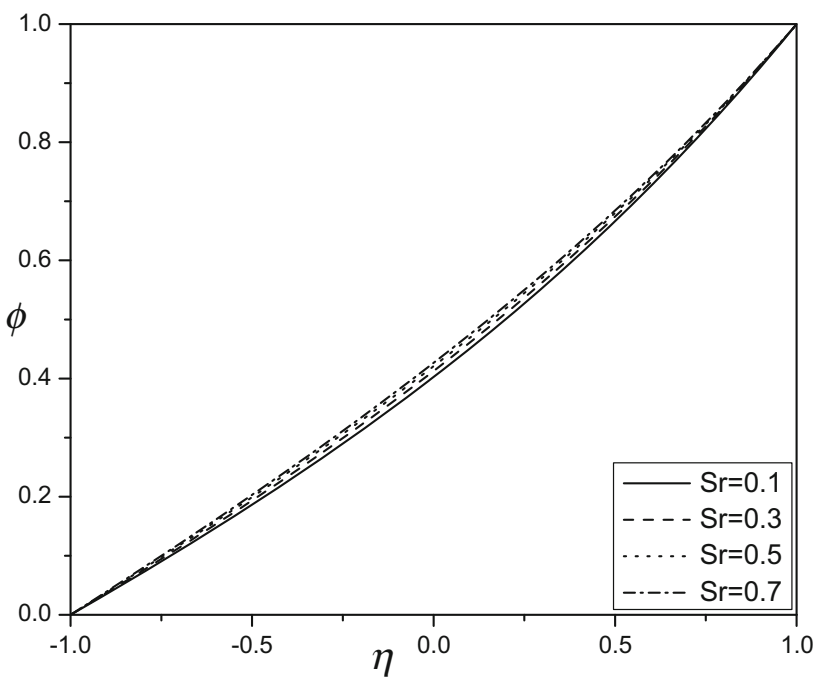

Fig. 15 Soret effect on $\phi(\eta)$ when $\beta_{1}=0.1, \beta_{2}=0.1, D_{f}=$ $0.5, K=1.0$

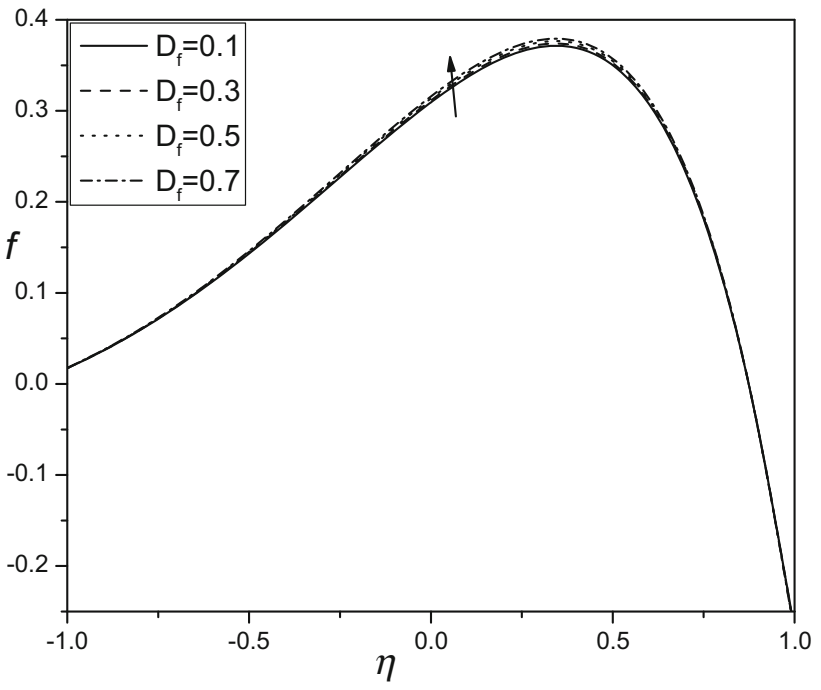

Fig. 16 Dufour effect on $f(\eta)$ when $\beta_{1}=0.1, \beta_{2}=0.1, S r=$ $0.5, K=1.0$

of the fluid increases with an increase in diffusion thermo parameter. Since the values of $D_{f}$ increases due to either an increase in the temperature difference or decrease in the concentration difference, i.e., the lowest peak of the flow velocity compatible with the lowest Dufour number. The effect of diffusion thermo parameter on dimensionless temperature is presented in Fig. 17. It is noted that the temperature profile increases with an increase in Dufour number. Figure 18 demonstrates that the concentration of the fluid decreases as Dufour effect increases. This is because of temperature gradients contribution to diffusion of the species. 


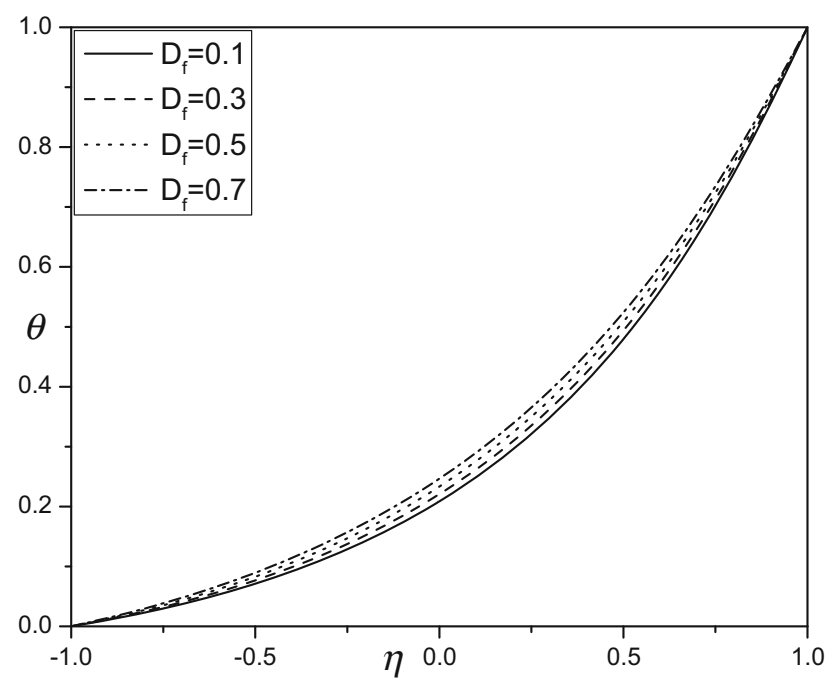

Fig. 17 Dufour effect on $\theta(\eta)$ when $\beta_{1}=0.1, \beta_{2}=0.1, S r=$ $0.5, K=1.0$

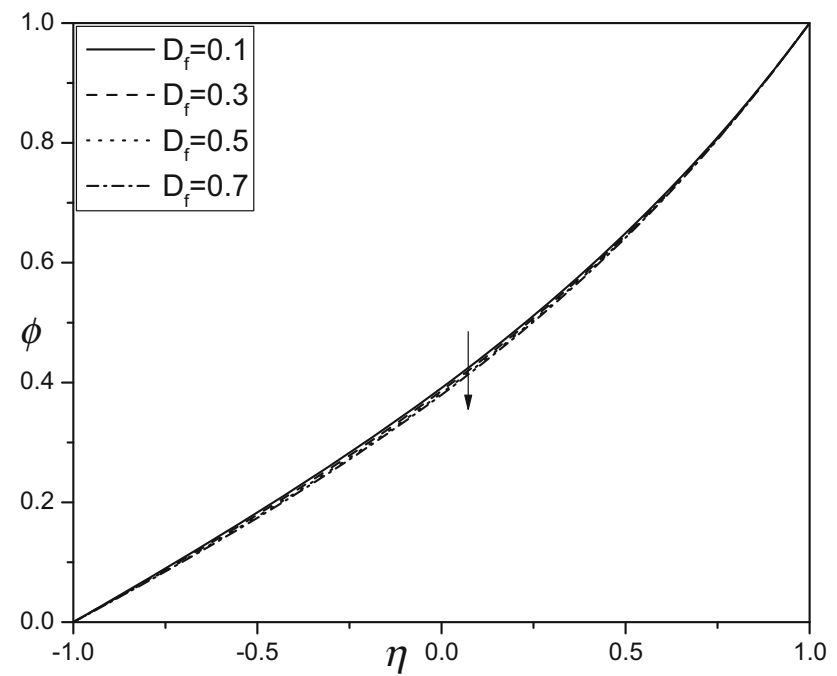

Fig. 18 Dufour effect on $\phi(\eta)$ when $\beta_{1}=0.1, \beta_{2}=0.1, S r=$ $0.5, K=1.0$

The chemical reaction parameter $K$ effect on velocity, temperature and concentration profiles are shown in Figs. 19, 20 and 21. It is observed from these figures that the fluid flow velocity decreases as $K$ increases. The temperature increases and concentration of the fluid decreases when the chemical reaction parameter increases. Since the chemical molecular diffusivity drop down when chemical reaction is high, i.e., lesser diffusion. Hence, they are procured by the transfer of species. The higher $K$ will decrease the concentration species. Therefore, with the increase in $K$ suppresses the distribution of the concentration at all the points of the fluid flow. With this, it can be

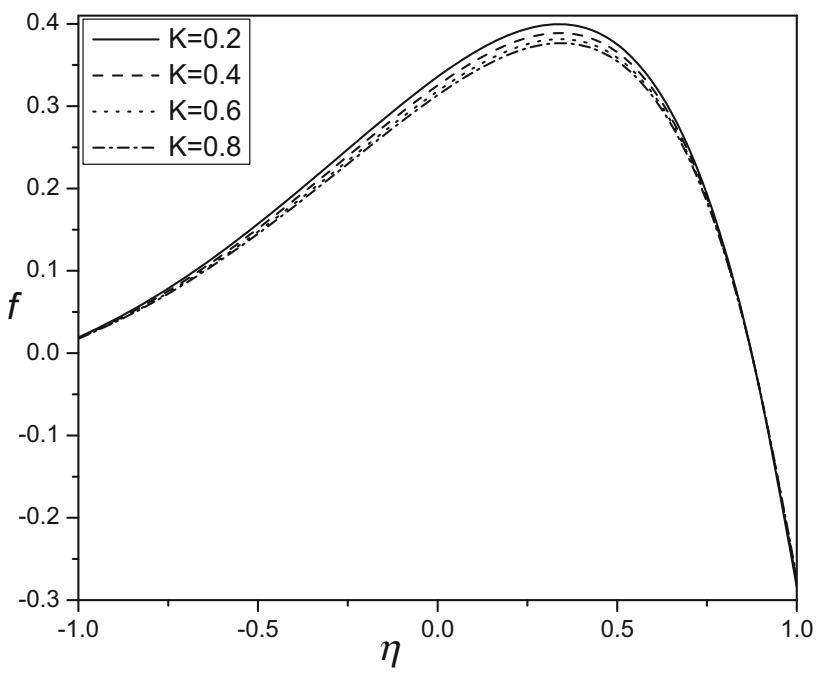

Fig. 19 Chemical reaction effect on $f(\eta)$ when $\beta_{1}=0.1, \beta_{2}=$ $0.1, S r=0.5, D_{f}=0.5$

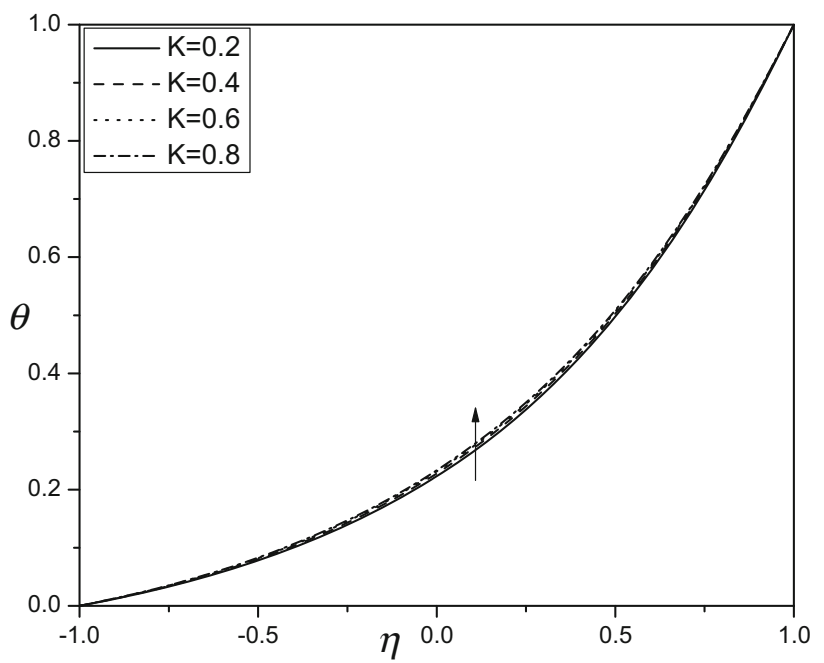

Fig. 20 Chemical reaction effect on $\theta(\eta)$ when $\beta_{1}=0.1, \beta_{2}=$ $0.1, S r=0.5, D_{f}=0.5$

claimed that on the distribution of the concentration, massive effect is higher with heavier diffusing species.

Variation of slip parameters $\left(\beta_{1}\right.$ and $\left.\beta_{2}\right)$, thermal diffusion parameter $(S r)$, diffusion thermo parameter $\left(D_{f}\right)$, together with the chemical reaction parameter $K$ are shown in Table 6 by fixing the remaining parameters. It is seen that the friction factor decreases at both the walls with an increase in the slip parameters $\beta_{1}$ and $\beta_{2}$. It is clear that increase in $\beta_{1}$ leads to increase in heat transfer coefficient at the plates where as the mass transfer rate increases at the initial wall and decreases at the terminal wall. It can be 
observed that at the initial plate the heat transfer rate decreases and at the second plate it is found to be increasing while the reverse trend is perceived on mass transfer rate with an increase in $\beta_{2}$. It is observed from this table that the skin friction coefficient, heat transfer rate increases at the injection wall and decreases at the terminal wall with the increase of Soret parameter $(\mathrm{Sr})$, where as mass transfer rate have reverse trend at both the plates. It is noticed that higher values of Dufour parameter $\left(D_{f}\right)$ increases the friction factor and mass transfer rate at the initial plate and decreases at the suction wall, while heat transfer rate shows the reverse trend at two walls. The effect of chemical reaction parameter $K$ on skin friction, heat and mass transfer rates are presented in Table 6. It is

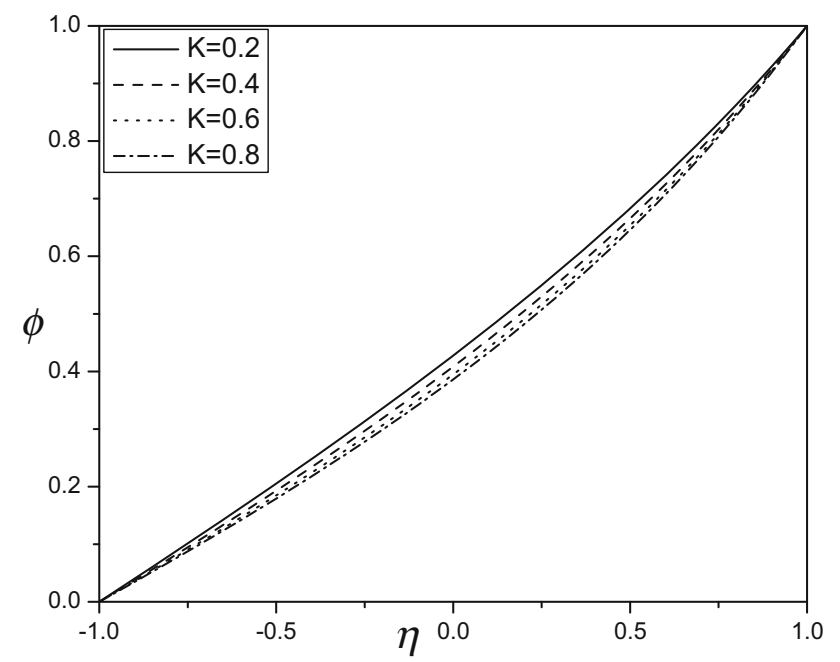

Fig. 21 Chemical reaction effect on $\phi(\eta)$ when $\beta_{1}=0.1, \beta_{2}=0.1, S r=0.5, D_{f}=0.5$ seen from the table that all the physical parameters are decreasing at the injection wall and those are increasing at the suction wall with the enhance in $K$. It is also seen from this table that the higher values of $K$ increases the mass transfer rate at $\eta=-1$ and decreases at $\eta=1$. The influence of the emerging parameters are patently obvious from the Table 6, and hence are not discussed for conciseness.

\section{Conclusions}

Two-dimensional natural convection flow in a vertical channel in the presence of chemical reaction and the cross diffusion effects with Navier slip. Homotopy analysis method has been employed to solve the final system of equations. The markable findings are summarized as:

- Flow velocity and the rate of mass transfer increases at the injection wall, decreases slightly at the suction wall and the rate of heat transfer increases at both the walls with an enhance in slip parameter $\beta_{1}$.

- As $\beta_{2}$ increases, the fluid flow velocity and friction factors decrease (slightly at the injection wall and marginally at the suction wall). Where as heat transfer rate disintegrates at the injection wall and increases at the end wall, while the opposite nature is noticed on the rate of mass transfer with an enhance in $\beta_{2}$.

- It is noticed that the velocity and concentration of the fluid increases and temperature profile decreases with an increase in Soret parameter. In addition, it is identified that the friction factor, Nusselt number increases at the injection plate and decreases at the end wall as $\mathrm{Sr}$ increases, where as mass transfer rate shows the reverse trend at both the walls.
Table 6 Effects of skin friction coefficient, heat and mass transfer rates for various values of $S r, D_{f}, \beta_{1}, \beta_{2}$ and $K$

\begin{tabular}{lllllllllll}
\hline$\beta_{1}$ & $\beta_{2}$ & $S r$ & $D_{f}$ & $K$ & $f^{\prime}(-1)$ & $f^{\prime}(1)$ & $N u_{1}$ & $N u_{1}$ & $S h_{1}$ & $S h_{2}$ \\
\hline 0.1 & 0.1 & 0.5 & 0.5 & 0.5 & 0.1829 & -2.7919 & -0.1154 & -1.2373 & -0.3733 & -0.7861 \\
0.2 & 0.1 & 0.5 & 0.5 & 0.5 & 0.1820 & -2.8385 & -0.1153 & -1.2317 & -0.3732 & -0.7866 \\
0.3 & 0.1 & 0.5 & 0.5 & 0.5 & 0.1811 & -2.8846 & -0.1152 & -1.2261 & -0.3731 & -0.7871 \\
0.1 & 0.1 & 0.5 & 0.5 & 0.5 & 0.1829 & -2.7919 & -0.1154 & -1.2373 & -0.3733 & -0.7861 \\
0.1 & 0.2 & 0.5 & 0.5 & 0.5 & 0.1651 & -3.7536 & -0.1162 & -1.0957 & -0.3709 & -0.7990 \\
0.1 & 0.3 & 0.5 & 0.5 & 0.5 & 0.1287 & -5.7585 & -0.1252 & -0.6181 & -0.3600 & -0.8402 \\
0.1 & 0.1 & 0.2 & 0.5 & 0.5 & 0.1762 & -2.7462 & -0.1191 & -1.2214 & -0.3476 & -0.8326 \\
0.1 & 0.1 & 0.4 & 0.5 & 0.5 & 0.1806 & -2.7765 & -0.1166 & -1.2319 & -0.3646 & -0.8018 \\
0.1 & 0.1 & 0.6 & 0.5 & 0.5 & 0.1852 & -2.8076 & -0.1140 & -1.2427 & -0.3819 & -0.7701 \\
0.1 & 0.1 & 0.5 & 0.2 & 0.5 & 0.1817 & -2.7756 & -0.1028 & -1.3161 & -0.3792 & -0.7824 \\
0.1 & 0.1 & 0.5 & 0.4 & 0.5 & 0.1825 & -2.7863 & -0.1110 & -1.2639 & -0.3753 & -0.7849 \\
0.1 & 0.1 & 0.5 & 0.6 & 0.5 & 0.1833 & -2.7977 & -0.1198 & -1.2104 & -0.3711 & -0.7873 \\
0.1 & 0.1 & 0.5 & 0.5 & 0.2 & 0.1882 & -2.8287 & -0.1127 & -1.2507 & -0.3924 & -0.7466 \\
0.1 & 0.1 & 0.5 & 0.5 & 0.4 & 0.1846 & -2.8040 & -0.1145 & -1.2417 & -0.3795 & -0.7731 \\
0.1 & 0.1 & 0.5 & 0.5 & 0.6 & 0.1812 & -2.7801 & -0.1161 & -1.2329 & -0.3671 & -0.7989 \\
\hline
\end{tabular}


- Fluid flow velocity, $\theta$ increases and the dimensionless concentration profile decreases as $D_{f}$ increases. It is seen that as Dufour parameter increases, the skin friction and mass transfer rate at $\eta=-1$ and decreases at $\eta=1$ wall, while the reverse trend is noticed in case of heat transfer rate at both the walls.

- As reaction parameter increases, the velocity and concentration of the fluid decreases and the temperature profile decreases. It is also observed that the values of $f^{\prime}(0)$, Nusselt and Sherwood numbers decrease at the initial wall and those increase at the terminal plate with the increase in $\mathrm{K}$.

Open Access This article is distributed under the terms of the Creative Commons Attribution 4.0 International License (http://crea tivecommons.org/licenses/by/4.0/), which permits unrestricted use, distribution, and reproduction in any medium, provided you give appropriate credit to the original author(s) and the source, provide a link to the Creative Commons license, and indicate if changes were made.

\section{References}

1. Kairi, R.R., Murthy, P.: Effect of viscous dissipation on natural convection heat and mass transfer from vertical cone in a nonNewtonian fluid saturated non-Darcy porous medium. Appl. Math. Comput. 217(20), 8100-8114 (2011)

2. Fattahi, E., Farhadi, M., Sedighi, K., Nemati, H.: Lattice Boltzmann simulation of natural convection heat transfer in nanofluids. Int. J. Therm. Sci. 52, 137-144 (2012)

3. Huelsz, G., Rechtman, R.: Heat transfer due to natural convection in an inclined square cavity using the lattice Boltzmann equation method. Int. J. Therm. Sci. 65, 111-119 (2013)

4. Terekhov, V.I., Ekaid, A.L., Yassin, K.F.: Laminar free convection heat transfer between vertical isothermal plates. J. Eng. Thermophys. 25(4), 509-519 (2016)

5. Navier, C.L.M.H.: Memoire sur les lois du mouvement des fluides. Mem. Acad. R. Sci. Paris 6, 389-416 (1823)

6. Eegunjobi, A.S., Makinde, O.D.: Effects of the slip boundary condition on non-Newtonian flows in a channel. Entropy 14(12), 1028-1044 (2012)

7. Rundora, L., Makinde, O.D.: Effects of Navier slip on unsteady flow of a reactive variable viscosity non-Newtonian fluid through a porous saturated medium with asymmetric convective boundary conditions. J. Hydrodyn. Ser. B. 27(6), 934-944 (2015)

8. Ng, C.-O.: Starting flow in channels with boundary slip. Meccanica. doi:10.1007/s11012-016-0384-4

9. Mahmoud, M.A.A., Megahed, A.M.: Thermal radiation effect on mixed convection heat and mass transfer of a non-Newtonian fluid over a vertical surface embedded in a porous medium in the presence of thermal diffusion and diffusion-thermo effects. J. Appl. Mech. Tech. Phys. 54(1), 90-99 (2013)

10. Srinivasacharya, D., Mallikarjuna, B., Bhuvanavijaya, R.: Soret and Dufour effects on mixed convection along a vertical wavy surface in a porous medium with variable properties. Ain Shams Eng. J. 6(2), 553-564 (2015)

11. Umar, K., Naveed, A., Tauseef, S., Mohyud-Din.: Soret and Dufour effects on flow in converging and diverging channels with chemical reaction. Aerospace Sci. Technol. 49, 135-143 (2016)

12. Kothandapani, M., Prakash, J.: Effects of thermal radiation and chemical reactions on peristaltic flow of a Newtonian nanofluid under inclined magnetic field in a generalized vertical channel using homotopy perturbation method. Asia-Pac. J. Chem. Eng. 10(2), 259-272 (2015)

13. RamReddy, C., Pradeepa, T.: Spectral quasi-linearization method for homogeneous-heterogeneous reactions on nonlinear convection flow of micropolar fluid saturated porous medium with convective boundary condition. Open Eng. 6(1), 106-119 (2016)

14. Liao, S.: Beyond perturbation. Introduction to homotopy analysis method. Chapman and Hall/CRC Press, Boca Raton (2003)

15. Liao, S.: An optimal homotopy-analysis approach for strongly nonlinear differential equations. Commun. Nonlinear Sci. Numer. Simul. 15(8), 2003-2016 (2010)

16. Srinivasacharya, D., Kaladhar, K.: Mixed convection flow of couple stress fluid between parallel vertical plates with Hall and Ion-slip effects. Commun. Nonlinear Sci. Numer. Simul. 17(6), 2447-2462 (2012)

17. Abbasbandy, S., Shivanian, E.: Solution of singular linear vibrational BVPs by the homotopy analysis method. J. Numer. Math. Stoch. 1(1), 77-84 (2009)

18. Abbasbandy, S., Shivanian, E., Vajravelu, K.: Mathematical properties of h-curve in the frame work of the homotopy analysis method. Commun. Nonlinear Sci. Numer. Simul. 16(11), 4268-4275 (2011)

19. Ellahi, R., Shivanian, E., Abbasbandy, S., Rahman, S.U., Hayat, T.: Analysis of steady flows in viscous fluid with heat/mass transfer and slip effects. Int. J. Heat Mass Transfer. 55(23), 6384-6390 (2012)

20. Shaban, M., Shivanian, E., Abbasbandy, S.: Analyzing magnetohydrodynamic squeezing flow between two parallel disks with suction or injection by a new hybrid method based on the Tau method and the homotopy analysis method. Eur. Phys. J. Plus. 128(11), 1-10 (2013)

21. Shivanian, E., Abbasbandy, S.: Predictor homotopy analysis method: two points second order boundary value problems. Nonlinear Anal. Real World Appl. 15, 89-99 (2014)

22. Ahmad Soltani, L., Shivanian, E., Ezzati, R.: Convection-radiation heat transfer in solar heat exchangers filled with a porous medium: exact and shooting homotopy analysis solution. Appl. Therm. Eng. 103, 537-542 (2016)

23. Ellahi, R., Shivanian, E., Abbasbandy, S., Hayat, T.: Numerical study of magnetohydrodynamics generalized Couette flow of Eyring-Powell fluid with heat transfer and slip condition. Int. J. Numer. Methods Heat Fluid Flow 26(5), 1433-1445 (2016)

24. Rashidi, M.M., Mohimanian pour, S.A., Abbasbandy, S.: Analytic approximate solutions for heat transfer of a micropolar fluid through a porous medium with radiation. Commun. Nonlinear Sci. Numer. Simul. 16, 1874-1889 (2011)

25. Turkyilmazoglu, M.: Numerical and analytical solutions for the flow and heat transfer near the equator of an MHD boundary layer over a porous rotating sphere. Int. J. Therm. Sci. 50, 831-842 (2011) 